\title{
Impact of chlorogenic acids from coffee on urine metabolome in healthy human subjects
}

\author{
Francisco Madrid-Gambin ${ }^{\mathrm{a}}$, Mar Garcia-Aloy ${ }^{\mathrm{a}, 1}$, Rosa Vázquez-Fresno ${ }^{\mathrm{a}, 1, *}$, Esteban \\ Vegas-Lozano $^{\mathrm{b}}$, M. Carmen Ruiz de Villa Jubany ${ }^{\mathrm{b}}$, Koichi Misawa ${ }^{\mathrm{c}}$, Tadashi Hase ${ }^{\mathrm{c}}$, \\ Akira Shimotoyodome ${ }^{c}$, Cristina Andres-Lacueva ${ }^{\mathrm{a},{ }^{*}}$
}

\begin{abstract}
aBiomarkers \& Nutrimetabolomic Lab., Nutrition and Food Science Department, XaRTA, INSA, Campus Torribera, Pharmacy Faculty, University of Barcelona, Barcelona, Spain.

${ }^{b}$ Department of Statistics, University of Barcelona, Barcelona, Spain

${ }^{c}$ Biological Science Laboratories, Kao Corporation, 2606, Akabane, Ichikai, Haga, Tochigi 321-3497, Japan

${ }^{1}$ These authors contributed equally to the work.

*Correspondence: Rosa Vázquez-Fresno and Cristina Andres-Lacueva. Biomarkers \& Nutrimetabolomic Lab., Nutrition and Food Science Department, Campus Torribera, Pharmacy Faculty, University of Barcelona, Av. Joan XXIII s/n, 08028 Barcelona, Spain. E-mail: candres@ub.edu and rosavfr@gmail.com.
\end{abstract}

\section{HIGHLIGHTS}

- Acute intake of CGAs from coffee impacts on central energetic metabolism.

- Sustained intake of CGAs from coffee plays a role in microbiota-derived compounds.

- Trigonelline is a coffee extract beverage biomarker in short- and long-term.

- Direct excretion of trigonelline would avoid interindividual variation.

- The use of uni- and multivariate analyses reinforced the biological effects.

\begin{abstract}
Several studies suggest that coffee has some benefits for health; however, little is known about the specific role of the main polyphenol compounds of coffee, chlorogenic acids (CGAs), without caffeine interaction. A ${ }^{1} \mathrm{H}-\mathrm{Nuclear}$ Magnetic Resonance ( $\left.{ }^{1} \mathrm{H}-\mathrm{NMR}\right)$-based metabolomics approach was used to assess the effect of CGAs from coffee on the human urine metabolome. Ten male volunteers participated in a dietary crossover randomized intervention study with a rich CGAs coffee extract beverage (CEB: $223 \mathrm{mg} / 100 \mathrm{ml}$ of CGAs). The study consisted of a daily intake of CEB or a control beverage with equal caffeine dose during 28 days. Fasting urines collected at the first and last days of each period of the study were analyzed using an CGAs untargeted ${ }^{1} \mathrm{H}-\mathrm{NMR}$ approach. Additionally, 4-hour postpandrial urines after the first intake of each beverage were also analyzed. Uni- and multi-variate statistic approaches
\end{abstract}


were used to strengthen the results. Multilevel partial least squares discriminant analysis (MLPLS-DA) was used to paired comparisons across the crossover design. A further univariate analysis model for crossover studies was performed to assess the significant changes. Acute consumption of CEB resulted in high excretion of 2-furoylglycine, likewise endogenous compounds such as succinic, citric, 3-methyl-2-oxovaleric and isobutyric acids. Sustained consumption of CEB showed an increase of microbiota-derived compounds such as hippuric, 3-(3-Hydroxyphenyl)-3-hydroxypropionic and 3-hydroxyhippuric acids in urine. Moreover, trigonelline was found in urine after both acute and sustained intakes, as well as in the composition of the beverage exhibiting a direct excretion of this biomarker without any biotransformation, suggesting a non-interindividual variation.

Abbreviations: CEB, coffee extract beverage; CGAs, chlorogenic acids; FID, free induction decay; HPHPA, 3-(3-Hydroxyphenyl)-3-hydroxypropionic acid; LR, loading rank; ML-PLSDA, multilevel partial least squares discriminant analysis; NMP, N-methylpyridinium; NMR, Nuclear Magnetic Resonance; PCA, principal component analysis; TCA, tricarboxylic acid; TSP, 3-(trimethylsilyl)-proprionate-2,2,3,3-d4

Keywords: Coffee; Chlorogenic acids; Metabolomics; NMR; Biomarkers; Multilevel PLS-DA

\section{INTRODUCTION}

Coffee is one of the most widely consumed beverages worldwide, and therefore there are a huge number of studies concerning its benefits for health (Cowan, T. E., et al., 2014; Guertin, K. a, et al., 2015; R M van Dam \& Hu, 2005). Coffee beans contain a large variety of biologically active compounds, the predominant ones being chlorogenic acids (CGAs) and caffeine (Johnston, Clifford, \& Morgan, 2003). Several epidemiological researches suggest that coffee consumption may help prevent chronic diseases, including type 2 diabetes mellitus (Van Dam \& Feskens, 2002), obesity and metabolic syndrome (Nordestgaard, Thomsen, \& Nordestgaard, 2015), Parkinson's disease (Sääksjärvi et al., 2008) and liver disease (La Vecchia, 2005). Moreover, coffee is rich in many polyphenols and diterpenes (Urgert \& Katan, 1997), which have antioxidant properties (Yanagimoto, Ochi, Lee, \& Shibamoto, 2004) and may also mitigate harmful gut microbiota species with its regular consumption (Cowan et al., 2014). Although these beneficial effects are attributed to caffeinated coffee, high intakes of decaffeinated coffee have also been associated with a reduced risk of type 2 diabetes mellitus (Ding, M., et al., 2014; Huxley, R., et al., 2009) and positive effects on cognition and 
psychomotor behavior (Shukitt-Hale, Miller, Chu, Lyle, \& Joseph, 2013), indicating that some compounds in coffee other than caffeine may have a protective effect. Few interventional studies focused on the effect of bioactive compounds in coffee in both the short- and long-term have been able to provide an explanation for physiological mechanisms. However, in most epidemiological studies there is no distinction between caffeinated coffee consumers and newer or occasional decaffeinated coffee consumers (Higdon \& Frei, 2006). Therefore the role of CGAs alone in this beverage is still unclear.

The application of metabolomics in the nutrition field can provide new information on dietary components, with the discovery of new biomarkers of food exposure, thereby revealing potential modifications in diet-related pathways in early disease stages likewise in healthy individuals (Puiggròs, F., et al., 2015; Scalbert, A., et al., 2014). Nuclear Magnetic Resonance (NMR) in metabolomics is a fast, robust, reproducible, non-destructive technique (Wishart, 2008). Metabolomics allows a global description of metabolites that gives detailed information on metabolic pathways and in turn on biological processes, thereby clarifying associations with health benefits and elucidating underlying mechanisms (Brennan, 2014). Untargeted metabolomics is generally used for the discovery of metabolites and modifications in global metabolic pathways, whereas targeted metabolomics is focused on specific metabolic pathways (Xu, Wang, Ho, \& Ong, 2014).

Several biomarkers of coffee intake have previously been proposed, including trigonelline, 2furoylglycine, caffeine and several polyphenols (Heinzmann, S. S., et al., 2015; Ito, H., et al., 2005; Lang, R., et al., 2011; Lloyd, A. J., et al., 2013; Rothwell, J. A., et al., 2014; Stalmach, A., et al., 2009). Coffee is the main dietary source of CGAs (Clifford, 2000), but although CGAs and caffeic acid are the main phenolic compounds identified in urine after coffee intake in shortterm (Ito et al., 2005), derived compounds of polyphenol metabolism are dependent on high interindividual variation in humans in long-term because of gut microbiota (Hervert-Hernández \& Goñi, 2011).

The present study was conducted to determine whether CGAs from coffee may impact on the human urine metabolome through an untargeted metabolomic approach, and to identify the changes on the metabolome after both acute and sustained consumptions on healthy human volunteers. 


\section{MATERIAL AND METHODS}

\subsection{Subjects and study design}

Ten healthy male between 25 and 44 years old with a body mass index of $23.4 \pm 2.1 \mathrm{~kg} / \mathrm{m} 2$ (mean $\pm \mathrm{SD}$ ) participated in a randomized, double-blind, placebo-controlled, crossover clinical trial. Exclusion criteria included caffeine intoxication, intake of CGAs supplements, serious illness (such as heart disease, kidney disease or diabetes) and food allergies. The study protocol was approved by the Human Research Ethics Committee of Biological Science Laboratories of the KAO Corporation (ref: 507-20131218). This clinical trial was registered as International Standard Randomized Controlled Trial Number 15516017.

During the washout and the study periods, the subjects were forbidden to consume coffee and tea beverages other than test drink. Subjects were not allowed to consume alcoholic beverages and to practice exercise from 2 days before the beginning of the intervention. After a 14-day washout period, subjects were asked to consume a coffee extract beverage (CEB) containing $223 \mathrm{mg} / 100 \mathrm{ml}$ of CGAs (KAO Corporation, Japan) or a control beverage (caffeine-containing beverage). The daily dose of caffeine was similar between the intervention and control groups (see Table S1, Supporting information). Then, the participants consumed the corresponding beverage every day for the next 28 days (period I). The same procedure was repeated switching the individuals between the groups (period II) after a second 14-day washout period, in accordance with the crossover design. To analyze acute consumption, urine samples were collected during the first 4 postprandial hours after beverage intake on the first day of the intervention. For the analysis of sustained consumption, fasting urines on the first and last days of each period of the study were collected. All urine samples were stored in aliquots at $-80{ }^{\circ} \mathrm{C}$ prior to analysis.

\subsection{Sample preparation}

Both urine and beverage samples were thawed, vortexed and centrifuged at 13,200 rpm for 5 min. The supernatant $(600 \mu \mathrm{l})$ from each sample was mixed with an internal standard solution [120 $\mu$, consisting of $0.1 \%$ 3-(trimethylsilyl)-proprionate-2,2,3,3-d4 (TSP), chemical shift reference), $2 \mathrm{mM}$ of sodium azide (NaN3, bacteriostatic agent) and 1.5 M KH2PO4 in 99\% deuterium water (D2O)]. The optimized $\mathrm{pH}$ of the buffer was set at 7.0, with a potassium deuteroxide solution, to minimize variations in the chemical shifts of the NMR resonances. This mixture was transferred to a $5 \mathrm{~mm}$ NMR tube. 


\section{3. ${ }^{1} \mathrm{H}-\mathrm{NMR}$ data acquisition and processing}

The ${ }^{1} \mathrm{H}-\mathrm{NMR}$ urinary spectra were acquired on a Varian-Inova-500 MHz NMR spectrometer with presaturation of the water resonance using a NOESYPRESAT pulse sequence. During the acquisition, the internal temperature was kept constant at $298 \mathrm{~K}$. An exponential window function was applied to the free induction decay (FID) with a line-broadening factor of $0.3 \mathrm{~Hz}$ prior to the Fourier transformation. For each sample, FIDs were collected into $32 \mathrm{~K}$ data points, 128 scans, with a spectral width of $14 \mathrm{ppm}$, an acquisition time of $2 \mathrm{~s}$, a relaxation delay of $5 \mathrm{~s}$ and a mixing time of 100 ms (Vázquez-Fresno et al., 2012).

A CEB sample was acquired on a Bruker Avance III $400 \mathrm{MHz}$ NMR spectrometer equipped with a cryoprobe with presaturation of the water resonance using a NOESYPRESAT pulse sequence. The internal temperature was kept constant at $298 \mathrm{~K}$ and each sample was processed with a line-broadening factor of $0.3 \mathrm{~Hz}, 64$ scans with a spectral width of $15 \mathrm{ppm}$, an acquisition time of $3 \mathrm{~s}$, a relaxation delay of $5 \mathrm{~s}$ and a mixing time of $10 \mathrm{~ms}$.

NMR spectra were phased, baseline corrected and referenced (TSP, $0.0 \mathrm{ppm}$ ) using TopSpin software (version 3.0, Bruker, BioSpin, Germany). After baseline correction, original spectral data were bucketed in intelligent bucketing domains of $0.005 \mathrm{ppm}$ with ACD/NMR Processor 12.0 software (Advanced Chemistry Development, Toronto, Canada). The water signal and the regions above $9.5 \mathrm{ppm}$ and below $0.5 \mathrm{ppm}$ were excluded from the analysis.

\subsection{Data pre-processing and statistical analysis}

Data from acute and sustained interventions were submitted individually to MetaboAnalyst 3.0 for filtering and normalization purposes (Xia, Sinelnikov, Han, \& Wishart, 2015). To exclude data points showing little variance across experimental conditions, the matrix was interquartile range filtered. Data were row-wise normalized (rows were samples) by the sum of the intensities of the spectra and column-wise normalized (columns were metabolites) using Pareto scaling and cube root transformation. Both data sets were used for further statistical analyses.

All the statistical analyses were performed with $\mathrm{R}$ version 3.1.2. Principal component analysis (PCA) was performed to detect the presence of outliers and to evaluate a potential carryover effect. Multilevel partial least squares discriminant analysis (ML-PLS-DA) was used to paired comparisons of the effects of $\mathrm{CEB}$ versus control beverage exploiting the crossover design with 
the R package mixOmics (Lê Cao, González, \& Déjean, 2009). ML-PLS-DA is an extension of ordinary PLS-DA described by Van Velzen et al. (2008) which allows separation of the withinsubject variation from the inter-subject variation that could obscure nutrition-related metabolic effects. A "leave-one-subject-out" cross-validation was performed to assess the ML-PLS-DA model and the classification error rate was determined by comparing the predicted class with the original one. Discriminant variables were determined based on the minimum value of the classification error rate and also on the absolute value of loading scores from the first latent variable. Loading scores were ranked and the top 5\% of total variables were selected as discriminant ones as long as this value was within the range of the minimum classification error rate. These variables were used to build a sparse ML-PLS-DA, and the corresponding score plot was drawn. On the sparse ML-PLS-DA, the signs were ranked based on the absolute values of their loading scores (named loading ranks, LRs), giving the lowest LR-values to signs that had the highest loading scores.

The statistical univariate crossover model (Jones, B. and Kenward, M. G., 2014; Senn, S, 2002) of differences between baseline and intervention data was performed between groups to assess the statistical significances. In addition to individual effect, period and sequence variables were included in the crossover model for further correction on potential carryover effects. Statistical significance was considered at a $\mathrm{p}$-value $<0.05$.

\subsection{Metabolite identification}

Metabolite identification was performed using Chenomx NMR Suite Professional Software package (version 8.1; Chenomx Inc., Edmonton, Canada) and by comparing NMR spectral data to those available in databases such as the Human Metabolome Database (http://www.hmdb.ca), the Biological Magnetic Resonance Data Bank (http://www.bmrb.wisc.edu) and the Madison Metabolomics Consortium Database (www.mmcd.nmrfam.wisc.edu), along with the existing NMR-based metabolomics literature. In addition, quantification of the beverage metabolites was performed with Chenomx using the methyl resonance of TSP as an internal standard. Peak fitting with reference to the TSP signal allowed quantification of absolute concentrations for the identified compounds. Further, a Pearson's correlation test was performed to test the correlation between signals of the same metabolite. 


\section{RESULTS AND DISCUSSION}

\section{1. ${ }^{1} \mathrm{H}-\mathrm{NMR}$ profiling of the coffee extract beverage}

In order to analyze the urine metabolites coming from the coffee beverage, metabolic profiling of the CEB was carried out. The identified and quantified metabolites are summarized in Table 1 and are shown on spectra in Fig. 1.

Table 1. Compounds identified by NMR spectroscopy in the coffee beverage (CEB) used in the present study.

\begin{tabular}{lc}
\hline Compound & $\mathrm{mg} / 100 \mathrm{ml}$ \\
\hline 2-Furoic acid & 0.56 \\
3-O-Caffeoylquinic acid & 48.02 \\
4-O-Caffeoylquinic acid & 47.03 \\
5-O-Caffeoylquinic acid & 44.23 \\
Acetic acid & 20.36 \\
Alanine & 0.61 \\
Caffeic acid & 0.43 \\
Caffeine & 22.65 \\
Choline & 2.08 \\
Citrate & 1.75 \\
Formic acid & 9.45 \\
Fumaric acid & 0.64 \\
Furfuryl alcohol & 2.63 \\
Lactic acid & 3.79 \\
Malic acid & 22.78 \\
Methanol & 0.20 \\
$N$-Methylpyridinium & $-\mathrm{a}$ \\
Nicotinic acid & 0.36 \\
Myo-inositol & 9.52 \\
Propionic acid & 1.03 \\
Sucrose & 10.22 \\
Quinic acid & 15.83 \\
Trigonelline & 18.83 \\
\hline - idnifid but &
\end{tabular}

a - : identified but not quantified. 


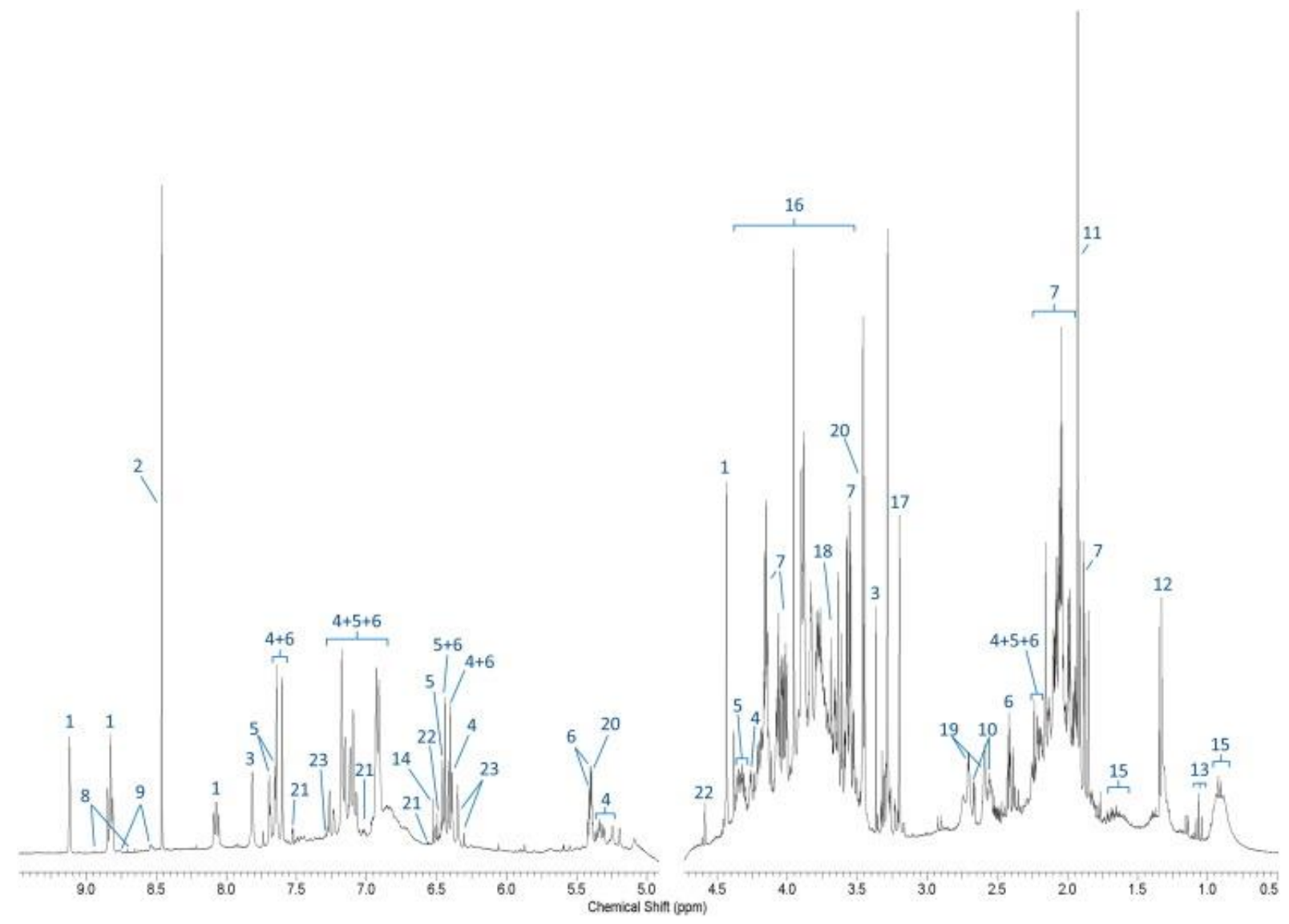

1, trigonelline; 2, formic acid; 3, caffeine; 4, 3-O-caffeoylquinic acid; 5, 4-O-caffeoylquinic acid; 6, 5-Ocaffeoylquinic acid; 7, quinic acid; 8, nicotinic acid; 9, n-methylpyridinium; 10, citric acid; 11, acetic acid; 12, lactic acid; 13, propionic acid; 14, fumaric acid; 15, lipids; 16, polysaccharides; 17, choline; 18, myo-inositol; 19, malic acid; 20, sucrose; 21, 2-furoic acid; 22, furfuryl alcohol; 23, caffeic acid.

Two furan compounds naturally present in coffee (2-furoic acid and furfuryl alcohol) were found in the CEB as precursors of 2-furoylglycine (Heinzmann et al., 2015), a compound found in urine after the acute intervention (see Section 3.4).

$\mathrm{N}$-methylpyridinium (NMP) and nicotinic acid, which were previously described in coffee by NMR ( Wei et al., 2012), were observed in the CEB but not detected in urine in this study. NMP and nicotinic acid are the main products of trigonelline degradation during pyrolysis and roasting (Stadler, Varga, Hau, Arce Vera, \& Welti, 2002). We assumed that the low concentration of NMP seen in the CEB (Fig. 1) would not be enough to detect this metabolite in urine samples in the present study, but it was observed in urine after coffee intake by other authors (Lang et al., 2011).

Caffeine, 3-O-caffeoylquinic, 4-O-caffeoylquinic and 5-O-caffeoylquinic, quinic and caffeic acids, which are common compounds in coffee ( Wei et al., 2012), were also found in the CEB. 


\subsection{Evaluation of carryover effects}

PCA was performed to explore the distribution of baseline samples according to the period (I and II) and the sequence (control $\rightarrow$ CEB; or CEB $\rightarrow$ control; Fig. S1) of the crossover study. A point cloud would indicate homogeneity at baseline. Fig. S1 shows that the sample of the period I from subject \#5 was outside of the point cloud and this may be due to a carryover effect. Therefore, all the statistical analyses were performed including and excluding subject \#5. This subject was included after ruling out biological differences in all analyses without any output variation. Accordingly, they did not indicate any carryover effect. However, additional correction for period and sequence was included in the univariate statistical crossover models.

\subsection{Uni- and multi-variate analyses}

Discriminant metabolites and chemical shifts identified with p-value and LR corresponding to uni- and multi-variate, respectively, are presented in Table 2. Fig. 3 presents box plots comparing the intensities of significant metabolites between CEB and control beverage.

The comparison of the differences between interventions resulted in ML-PLS-DA models with a minimum classification error rate of 0.1 and 0.0 for sustained and acute interventions, respectively (Fig. S2). The lowest rate was 0.1 for a range between the 6th and the 94 th variables after the sustained intervention, whereas the first discriminant variables after the acute intervention were strong enough for keeping the rate to 0 for all variables. The top $5 \%$ of loading scores were within the range of variables for these classification error rates. Therefore, two sparse ML-PLS-DA models were built with them. Fig. 2 presents the sparse ML-PLS-DAs which show a clear separation of treatments. The main discriminatory metabolites were projected in the first component and the contribution to treatment differences of each sign was set in LRs presented in Table 2. 
Table 2. Metabolites detected in urine after acute and sustained coffee extract beverage (CEB) in comparison with control beverage.

\begin{tabular}{|c|c|c|c|c|c|}
\hline $\begin{array}{l}\text { Intervention } \\
\text { type }\end{array}$ & Metabolite & $\delta$ (multiplicity) & $\begin{array}{l}\text { CBE vs. } \\
\text { control }\end{array}$ & $\mathrm{P}$ value ${ }^{\mathrm{a}}$ & $\mathrm{LR}^{\mathrm{b}}$ \\
\hline \multirow[t]{15}{*}{ Acute } & \multirow[t]{4}{*}{ Trigonelline } & $9.13(\mathrm{~s})$ & \multirow[t]{4}{*}{$\uparrow$} & $7.88 \times 10^{-8}$ & 4,5 \\
\hline & & 8.84 (d) & & $8.44 \times 10^{-7}$ & $10,11,14$ \\
\hline & & $8.08(\mathrm{t})$ & & $1.26 \times 10^{-2}$ & - \\
\hline & & $4.44(\mathrm{~s})$ & & $2.51 \times 10^{-6}$ & 15,25 \\
\hline & \multirow[t]{4}{*}{ 2-Furoylglycine } & $7.70(\mathrm{~s})$ & \multirow[t]{4}{*}{$\uparrow$} & $5.90 \times 10^{-4}$ & 39 \\
\hline & & $7.18(\mathrm{~d})$ & & $9.68 \times 10^{-2}$ & - \\
\hline & & $6.64(\mathrm{~m})$ & & $4.14 \times 10^{-2}$ & - \\
\hline & & $3.93(d)$ & & $3.00 \times 10^{-4}$ & 31,47 \\
\hline & \multirow[t]{2}{*}{ Citric acid } & $2.68(d)$ & \multirow[t]{2}{*}{$\uparrow$} & $8.72 \times 10^{-3}$ & - \\
\hline & & $2.54(\mathrm{~d})$ & & $1.31 \times 10^{-3}$ & 55 \\
\hline & Succinic acid & $2.41(\mathrm{~s})$ & $\uparrow$ & $7.78 \times 10^{-4}$ & 43 \\
\hline & 3-Methyl-2- & $1.10(\mathrm{~d})$ & $\uparrow$ & $1.35 \times 10^{-3}$ & 50 \\
\hline & oxovaleric acid & $0.90(\mathrm{t})$ & \multirow{3}{*}{$\uparrow$} & $1.30 \times 10^{-2}$ & - \\
\hline & \multirow[t]{2}{*}{ Isobutyric acid } & $1.07(d)$ & & $8.91 \times 10^{-3}$ & - \\
\hline & & $2.39(\mathrm{~m})$ & & $2.68 \times 10^{-2}$ & - \\
\hline \multirow[t]{20}{*}{ Sustained } & \multirow[t]{4}{*}{ Trigonelline } & $9.13(\mathrm{~s})$ & \multirow[t]{4}{*}{$\uparrow$} & $1.64 \times 10^{-3}$ & 8 \\
\hline & & $8.84(\mathrm{~d})$ & & $6.65 \times 10^{-4}$ & 7 \\
\hline & & $8.08(\mathrm{t})$ & & $9.54 \times 10^{-3}$ & - \\
\hline & & $4.44(\mathrm{~s})$ & & $9.98 \times 10^{-3}$ & 18 \\
\hline & \multirow[t]{5}{*}{ Hippuric acid } & $8.53(\mathrm{~s})$ & \multirow[t]{5}{*}{$\uparrow$} & $2.74 \times 10^{-3}$ & $11,17,24$ \\
\hline & & $7.84(\mathrm{~d})$ & & $7.30 \times 10^{-3}$ & - \\
\hline & & $7.64(\mathrm{t})$ & & $6.66 \times 10^{-5}$ & $2,5,10$ \\
\hline & & $7.56(\mathrm{t})$ & & $8.38 \times 10^{-3}$ & $28,34,56$ \\
\hline & & $3.97(d)$ & & $1.17 \times 10^{-2}$ & 22,35 \\
\hline & \multirow[t]{5}{*}{ HPHPA } & $7.31(\mathrm{t})$ & \multirow[t]{5}{*}{$\uparrow$} & $2.29 \times 10^{-4}$ & $1,3,27$ \\
\hline & & $6.98(d)$ & & $1.69 \times 10^{-2}$ & - \\
\hline & & $6.91(\mathrm{~s})$ & & $7.40 \times 10^{-3}$ & 44,47 \\
\hline & & $6.84(\mathrm{~d})$ & & $2.89 \times 10^{-2}$ & $16,21,49$ \\
\hline & & $5.03(\mathrm{~m}) \mathrm{c}$ & & - & - \\
\hline & \multirow{6}{*}{$\begin{array}{l}\text { 3-Hydroxyhippuric } \\
\text { acid }\end{array}$} & $7.40(\mathrm{t})$ & \multirow[t]{6}{*}{$\uparrow$} & $1.77 \times 10^{-2}$ & 55 \\
\hline & & $7.36(\mathrm{~s})$ & & $9.32 \times 10^{-4}$ & 6 \\
\hline & & $7.28(\mathrm{~s})$ & & $3.18 \times 10^{-1}$ & - \\
\hline & & $7.13(\mathrm{dd})$ & & $2.42 \times 10^{-2}$ & 42 \\
\hline & & $4.15(\mathrm{~d})$ & & $3.37 \times 10^{-2}$ & 60 \\
\hline & & $2.22(\mathrm{~s})$ & & $1.94 \times 10^{-2}$ & - \\
\hline
\end{tabular}

HPHPA: 3-(3-Hydroxyphenyl)-3-hydroxypropionic acid, s: singlet, d: doublet, t: triplet, dd: double doublet, m: multiplet.

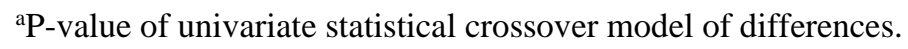

${ }^{b}$ Loading rank of ML-PLS-DA.

${ }^{\mathrm{c} S}$ Signal not considered because of proximity to water region. 
Fig. 2. Score plots of the subjects projected onto the first principal components, PC1 and PC2, after coffee extract beverage (CEB) in acute (A) and sustained (B) interventions.

A

Sparse ML-PLS-DA acute intervention

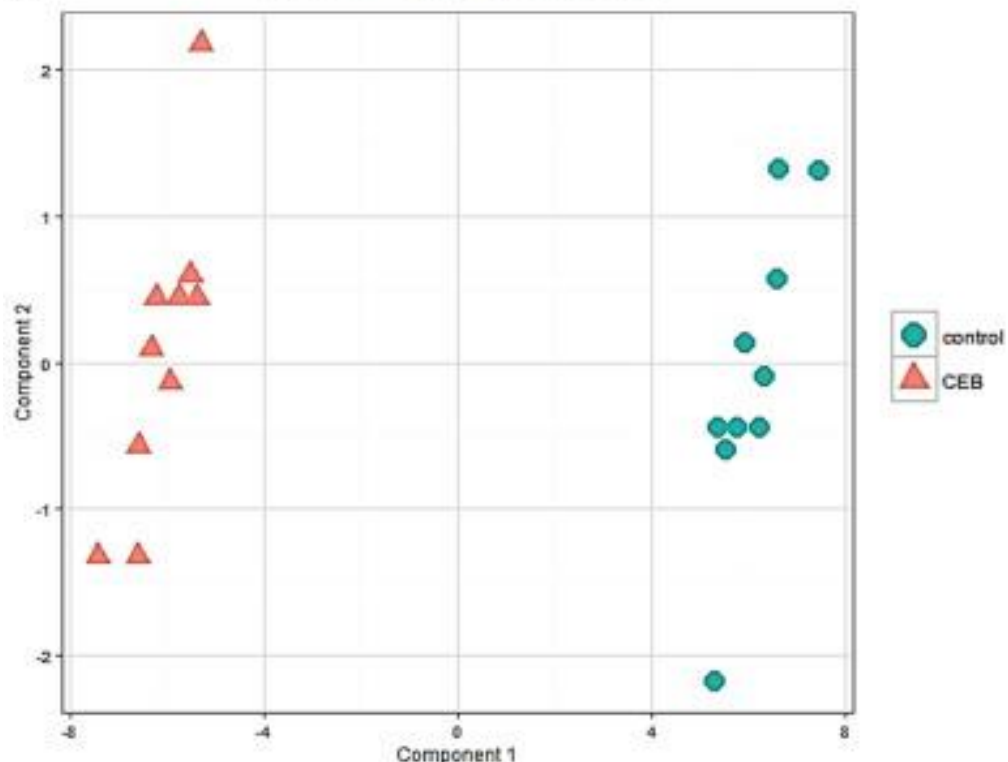

B

Sparse ML-PLS-DA sustained intervention

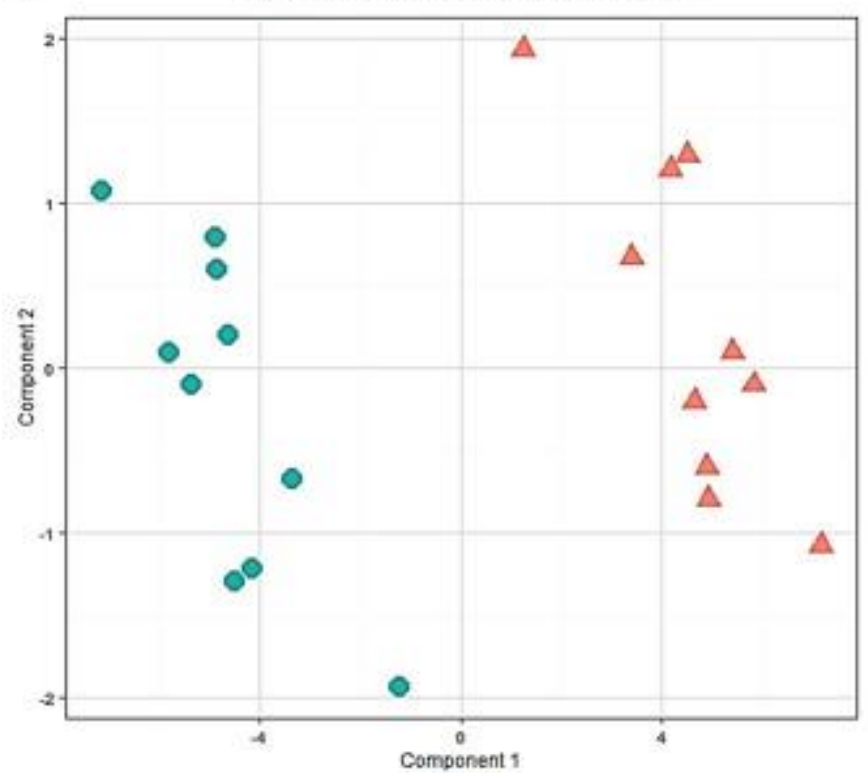

O control

$\triangle$ ces 
Fig. 3. Box plots of significant metabolites in urine after coffee extract beverage (CEB) in acute (A) and sustained (B) interventions.

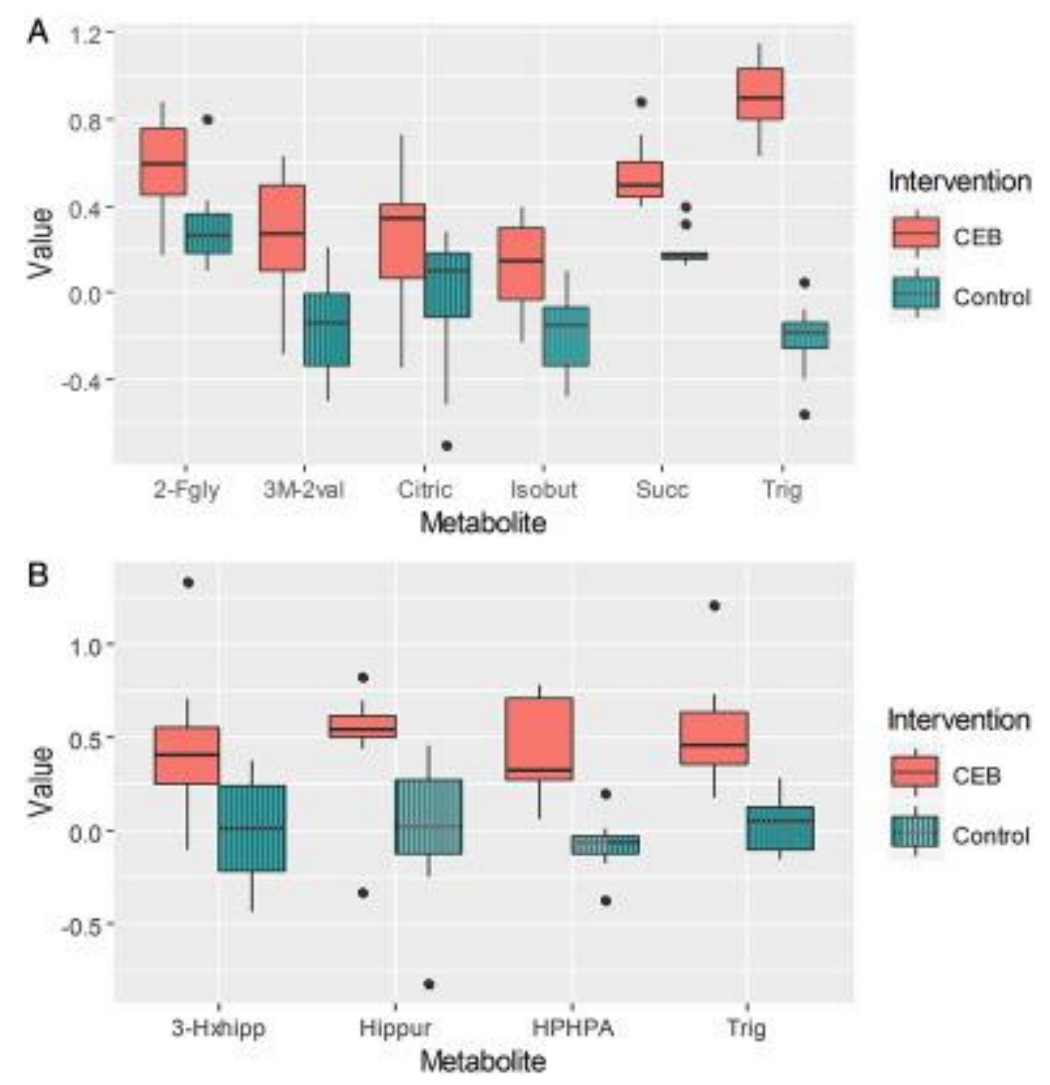

2-Fgly: 2-furoylglycine, 3M-2val: 3-methyl-2-oxovaleric acid, Citric: citric acid, Isobut: isobutyric acid, Succ: succinic acid, Trig: trigonelline, 3-Hxhipp: 3-hydroxyhippuric acid, Hippur: hippuric acid, HPHPA: 3-(3hydroxyphenyl)-3-hydroxypropionic acid.

\subsection{Acute intervention}

2-Furoylglycine observed after CBE intake is a product of furan derivatives contained in roasting products like coffee (Moon \& Shibamoto, 2009) or cocoa (Bonvehí, 2005) conjugated with glycine (Ginz, M. and Engelhardt, U. H., 2000 ; Pettersen, J. E. and Jellum, E., 1972). The furan product 2-furoylglycine has been identified after cocoa intake (Garcia-Aloy et al., 2015) and also associated with coffee intake in epidemiological studies (Guertin et al., 2014). Heinzmann and co-workers investigated its excretion kinetics, observing that maximal urinary excretion occurred $2 \mathrm{~h}$ after the coffee intake, returning to baseline levels after $24 \mathrm{~h}$ (Heinzmann et al., 2015). It explains its absence in the sustained intervention in the present study.

The increase of tricarboxylic acid (TCA) cycle intermediates suggests that CGAs coming from coffee may affect the central energy metabolism in humans. High levels of isobutyric and 3methyl-2-oxovaleric acids were observed. They are products of branched-chain amino acid 
(BCAA) catabolism: valine and isoleucine, respectively. Both valine (glucogenic BCAA) and isoleucine (ketogenic BCAA) are metabolized to succinic acid via the methylmalonyl-CoA (Hutson, Sweatt, \& LaNoue, 2005) TCA cycle, supported also by a significant higher excretion of succinic acid after CEB acute intake. In addition, an increase in the urinary level of citric acid was detected, which is also a TCA cycle intermediate, leading to the conclusion that CGAs coming from coffee may affect the central energy metabolism in humans, as was seen in a study with dietary supplementation with CGAs, which affected the circulating levels of intermediates of the TCA cycle in rats (Ruan, Yang, Zhou, Wen, et al., 2014). In accordance with energy metabolism, habitual coffee consumption was previously associated with weight control (Bakuradze et al., 2011) and a lower risk of type 2 diabetes (R M Van Dam, R. M. and Hu, F. B., 2005 ; Wedick, N. M., et al., 2011).

\subsection{Sustained intervention}

Hippuric, 3-hydroxyhippuric and 3-(3-Hydroxyphenyl)-3-hydroxypropionic (HPHPA) acids were detected after CEB in comparison with control beverage. These three compounds have been observed previously in epidemiological studies after coffee intake (Guertin et al., 2015). It has been suggested that CGAs are metabolized by microbiota in the lower gastrointestinal tract (Gonthier et al., 2006; M.-P. Gonthier, M.-P., et al., 2003 ; Ludwig, I. a., et al., 2013), obtaining as a product caffeic acid due to cleavage of the ester bond between caffeic and quinic acids by an esterase enzyme provided by the colonic microbiota (Plumb et al., 1999). The double bond of caffeic acid is reduced to form HPHPA prior to dihydroxylation of 3-(3,4dihydroxyphenyl)-propionic acid (Rechner et al., 2004). HPHPA may be absorbed from the colon to form hippuric and 3-hydroxyhippuric acids through $\beta$-oxidation and glycination in the liver (Rechner et al., 2002). Therefore, microbiota metabolites were downstream metabolites of 3-O-caffeoylquinic, 4-O-caffeoylquinic and 5-O-caffeoylquinic acids which were detected and identified in the CEB (see Section 3.1). Hippuric, 3-hydroxyhippuric and HPHPA acids are metabolites of microbiota fermentation of CGAs which are subsequently absorbed in the colon (Higdon \& Frei, 2006), playing a role in health effects (Williamson \& Clifford, 2010) and leading to the conclusion that CGAs may have an important function as precursors of microbiota-derived compounds observed after sustained consumption. 


\subsection{Common metabolites in urine and the coffee extract beverage}

Trigonelline was identified in urine after intake of the CEB in both acute and sustained interventions. According to the results, this metabolite comes from trigonelline naturally present in coffee beans (Beans, C., et al., 2009 ; Guertin, K. a, et al., 2015), as was observed in 1H-NMR analysis of the CEB (Fig. 1). Lang and co-workers observed this candidate biomarker up to three days after coffee consumption (Lang et al., 2011); therefore, trigonelline in the sustained intervention could correspond to trigonelline from the previous punctual intake of the day before. Thus, according to the results, trigonelline reflects coffee intake of both a punctual and a sustained consumption. This metabolite has been proposed as a candidate for dietary biomarker coffee intake (Lang, R., et al., 2011 ; Rothwell, J. A., et al., 2014). Trigonelline was also a common metabolite present in urine after both acute and sustained intakes as well as in the CEB, indicating a possible direct excretion (without any metabolism occurring) from coffee intake, as suggested by K. A. Guertin et al. (2015). A direct excretion of this metabolite suggests a non-interindividual variation in the metabolism of this compound, in contrast to microbiotaderived compounds of polyphenol metabolism, which are dependent on a high interindividual variation in humans (Hervert-Hernández \& Goñi, 2011). Hence, trigonelline seems to be a suitable biomarker of coffee intake after both acute and sustained interventions.

\section{CONCLUSIONS}

The results of the current study show the capability of an NMR-based metabolomic approach to detect significant changes in urinary metabolite profiling and help to clarify the role of specific coffee compounds other than caffeine in human metabolism after both acute and sustained interventions. Firstly, the increase of endogenous metabolites related to the TCA cycle such as succinic, citric, isobutyric and 3-methyl-2-oxovaleric acids after the acute study indicates that coffee CGAs impact on the central energy metabolism. Secondly, the present results exhibit two potential CEB biomarkers: 2-furoylglycine, after acute intake, and trigonelline, after both acute and sustained CEB intakes. Finally, there are other long-term metabolites after sustained intervention coming from the degradation of CGAs by gut microbiota observed through the identification of hippuric acid, 3-hydroxyhippuric acid and HPHPA. In conclusion, this study shows biomarkers of both short- and long-term intakes of coffee products along with other metabolites involved in energy metabolism (after acute intake) and microbiota metabolism (after sustained intake). 


\section{ACKNOWLEDGMENTS}

This research was funded by: the Fondo Europeo de Desarrollo Regional (FEDER) (AGL200913906-C02-01, AGL2010-10084-E) and the JPI HDHL FOODBALL (PCIN-2014-133MINECO-Spain). The clinical study was supported in part by the KAO Corporation and Fundació Bosch i Gimpera (FBG 307683). We also thank the award of 2014SGR1566 from the Generalitat de Catalunya's Agency AGAUR. FM acknowledges the APIF fellowship (University of Barcelona).

\section{REFERENCES}

Bakuradze, T., Boehm, N., Janzowski, C., Lang, R., Hofmann, T., Stockis, J. -P., ... Eisenbrand, G. (2011). Antioxidant-rich coffee reduces DNA damage, elevates glutathione status and contributes to weight control: Results from an intervention study. Molecular Nutrition \& Food Research, 55(5), 793-797. http://dx.doi.org/10.1002/mnfr.201100093.

Beans, C., Allred, K. F., Yackley, K. M., Vanamala, J., \& Allred, C. D. (2009). Trigonelline is a novel phytoestrogen in, 1833-1838. http://dx.doi.org/10.3945/jn.109.108001.1833.

Bonvehí, J. S. (2005). Investigation of aromatic compounds in roasted cocoa powder. European Food Research and Technology, 221(1-2), 19-29. http://dx.doi.org/10.1007/s00217-005-1147y.

Brennan, L. (2014). NMR-based metabolomics: From sample preparation to applications in nutrition research. Progress in Nuclear Magnetic Resonance Spectroscopy, 83, 42-49. http://dx.doi.org/10.1016/j.pnmrs.2014.09.001.

Clifford, M. N. (2000). Chlorogenic acids and other cinnamates - Nature, occurrence, dietary burden, absorption and metabolism. Journal of the Science of Food and Agriculture, 1043(October 1999), 1033-1043 (http://doi.org/http://dx.doi.org/10.1002/(SICI)10970010(20000515)80:7b1033::AID-JSFA595N3.0.CO;2-T).

Cowan, T. E., Palmnäs, M. S. a, Yang, J., Bomhof,M. R., Ardell, K. L., Reimer, R. a., ... Shearer, J. (2014). Chronic coffee consumption in the diet-induced obese rat: Impact on gut microbiota and serum metabolomics. Journal of Nutritional Biochemistry, 25(4), 489-495. http://dx.doi.org/10.1016/j.jnutbio.2013.12.009. 
Ding, M., Bhupathiraju, S. N., Chen, M., van Dam, R. M., \& Hu, F. B. (2014). Caffeinated and decaffeinated coffee consumption and risk of type 2 diabetes: A systematic review and a doseresponse meta-analysis. Diabetes Care, 37(2), 569-586. http://dx.doi.org/10.2337/dc13-1203.

Garcia-Aloy, M., Llorach, R., Urpi-Sarda, M., Auregui, O., Corella, D., Ruiz-Canela, M., ... Andres-Lacueva, C. (2015). A metabolomics-driven approach to predict cocoa product consumption by designing a multimetabolite biomarkermodel in free-living subjects from the PREDIMED study. Mol. Nutr. Food Research, 59, 212-220. http://dx.doi.org/10.1002/mnfr.201400434.

Ginz, M., \& Engelhardt, U. H. (2000). Identification of proline-based diketopiperazines in roasted coffee. Journal of Agricultural and Food Chemistry, 48(8), 3528-3532. http://dx.doi.org/10.1021/jf991256v.

Gonthier, M. P., Remesy, C., Scalbert, a, Cheynier, V., Souquet, J.M., Poutanen, K., \& Aura, a M. (2006).Microbialmetabolismof caffeic acid and its esters chlorogenic and caftaric acids by human faecal microbiota in vitro. Biomedicine and Pharmacotherapy, 60(9), 536-540. http://dx.doi.org/10.1016/j.biopha.2006.07.084.

Gonthier, M. -P., Verny, M. -A., Besson, C., Rémésy, C., \& Scalbert, A. (2003). Chlorogenic acid bioavailability largely depends on its metabolism by the gut microflora in rats. The Journal of Nutrition, 133(6), 1853-1859.

Guertin, K. a, Loftfield, E., Boca, S. M., Sampson, J. N., Moore, S. C., Xiao, Q., ... Sinha, R. (2015). Serum biomarkers of habitual coffee consumption may provide insight into the mechanism underlying the association between coffee consumption and colorectal cancerVols. $1-5$, (C). (pp. 1-12), 1-12. http://dx.doi.org/10.3945/ajcn.114.096099.

Guertin, K. A.,Moore, S. C., Sampson, J. N., Huang,W. -Y., Xiao, Q., Stolzenberg-Solomon, R. Z., ... Cross, A. J. (2014).Metabolomics in nutritional epidemiology: Identifyingmetabolites associated with diet and quantifying their potential to uncover diet-disease relations in populations. The American Journal of Clinical Nutrition, 100, 208-217. http://dx.doi.org/10.3945/ajcn.113.078758.

Heinzmann, S. S., Holmes, E., Kochhar, S., Nicholson, J. K., \& Schmitt-Kopplin, P. (2015). 2Furoylglycine as a candidate biomarker of coffee consumption. Journal of Agricultural and Food Chemistry, 63(38), 8615-8621. http://dx.doi.org/10.1021/acs.jafc.5b03040. 
Hervert-Hernández, D., \& Goñi, I. (2011). Dietary polyphenols and human gutmicrobiota: A review. Food Reviews International, 27(2), 154-169. http://dx.doi.org/10.1080/87559129.2010.535233.

Higdon, J., \& Frei, B. (2006). Coffee and health: A review of recent human research. Critical Reviews in Food Science and Nutrition, 46(2), 101-123. http://dx.doi.org/10.1080/10408390500400009.

Hutson, S. M., Sweatt, A. J., \& LaNoue, K. F. (2005). Branched-chain amino acid metabolism: Implications for establishing safe intakes. The Journal of Nutrition, 135(6),1557S-11564 Retrieved from http://jn.nutrition.org/cgi/content/long/135/6/1557S

Huxley, R., Lee, C. M. Y., Barzi, F., Timmermeister, L., Czernichow, S., Perkovic, V., ...Woodward, M. (2009). Coffee, decaffeinated coffee, and tea consumption in relation to incident type 2 diabetesmellitus: A systematic reviewwithmeta-analysis. Archives of Internal Medicine, 169(22), 2053-2063. http://dx.doi.org/10.1001/archinternmed.2009.439.

Ito, H., Gonthier, M. -P., Manach, C., Morand, C., Mennen, L., Rémésy, C., \& Scalbert, A. (2005). Polyphenol levels in human urine after intake of six different polyphenolrich beverages. The British Journal of Nutrition, 94, 500-509. http://dx.doi.org/10.1079/BJN20051522.

Johnston, K. L., Clifford,M. N., \&Morgan, L.M. (2003). Coffee acutely modifies gastrointestinal hormone secretion and glucose tolerance in humans: Glycemic effects of chlorogenic acid and caffeine. American Journal of Clinical Nutrition, 78(4), 728733.http://dx.doi.org/10.1021/jf980737w.

Jones, B., \& Kenward, M. G. (2014). Design and analysis of cross-over trials (3rd ed.). CRC Press Retrieved from https://books.google.com/books?id=aErNBQAAQBAJ\&pgis=1

La Vecchia, C. (2005). Coffee, liver enzymes, cirrhosis and liver cancer. Journal of Hepatology, 42(4), 444-446. http://dx.doi.org/10.1016/j.jhep.2005.01.004.

Lang, R., Wahl, A., Stark, T., \& Hofmann, T. (2011). Urinary N-methylpyridinium and trigonelline as candidate dietary biomarkers of coffee consumption. Molecular Nutrition and Food Research, 55(11), 1613-1623. http://dx.doi.org/10.1002/mnfr.201000656.

Lê Cao, K. -A., González, I., \& Déjean, S. (2009). integrOmics: An R package to unravel relationships between two omics datasets. Bioinformatics (Oxford, England), 25(21), 28552856. http://dx.doi.org/10.1093/bioinformatics/btp515. 
Lloyd, A. J., Beckmann, M., Haldar, S., Seal, C., Brandt, K., \& Draper, J. (2013). Data-driven strategy for the discovery of potential urinary biomarkers of habitual dietary exposure. American Journal of Clinical Nutrition, 97(C), 377-389. http://dx.doi.org/10.3945/ajen.112.048033.

Ludwig, I. a., Paz de Peña, M., Concepción, C., \& Alan, C. (2013). Catabolism of coffee chlorogenic acids by human colonic microbiota. BioFactors, 39(6), 623-632. http://dx.doi.org/10.1002/biof.1124.

Moon, J. -K., \& Shibamoto, T. (2009). Role of roasting conditions in the profile of volatile flavor chemicals formed from coffee beans. Journal of Agricultural and Food Chemistry, 57(13), 5823-5831 Retrieved from http://www.ncbi.nlm.nih.gov/pubmed/19579294

Nordestgaard, a. T., Thomsen, M., \& Nordestgaard, B. G. (2015). Coffee intake and risk of obesity, metabolic syndrome and type 2 diabetes: A Mendelian randomization study. International Journal of Epidemiology, 01, 1-15. http://dx.doi.org/10.1093/ije/dyv083.

Pettersen, J. E., \& Jellum, E. (1972). The identification and metabolic origin of 2-furoylglycine and 2.5-furandicarboxylic acid in human urine. Clinica Chimica Acta; International Journal of $\begin{array}{lllll}\text { Clinical } & \text { Chemistry, } & \text { 41, } & \text { 199-207 from }\end{array}$ http://www.ncbi.nlm.nih.gov/pubmed/4630229

Plumb, G. W., Garcia-Cones, M. T., Kroon, P. A., Rhodes, M., Ridley, S., \& Williams, G. (1999).Metabolismof chlorogenic acid by human plasma, liver, intestine and gutmicroflora 1. Journal of the Science of Food and Agriculture, 79, 390-392.

Puiggròs, F., Canela, N., \& Arola, L. (2015). Metabolome responses to physiological and nutritional challenges. Current opinion in food science. http://dx.doi.org/10.1016/j.cofs.2015.06.001.

Rechner, A. R., Kuhnle, G., Bremner, P., Hubbard, G. P., Moore, K. P., \& Rice-Evans, C. A. (2002). The metabolic fate of dietary polyphenols in humans. Free Radical Biology and Medicine, 33(2), 220-235. http://dx.doi.org/10.1016/S0891-5849(02)00877-8.

Rechner, A. R., Smith, M. A., Kuhnle, G., Gibson, G. R., Debnam, E. S., Srai, S. K. S., ... RiceEvans, C. A. (2004). Colonicmetabolism of dietary polyphenols: Influence of structure on microbial fermentation products. Free Radical Biology and Medicine, 36(2), 212-225. http://dx.doi.org/10.1016/j.freeradbiomed.2003.09.022. 
Rothwell, J. A., Fillâtre, Y., Martin, J. -F., Lyan, B., Pujos-Guillot, E., Fezeu, L., ... Manach, C. (2014). New biomarkers of coffee consumption identified by the non-targeted metabolomic profiling of cohort study subjects. PloS One, 9(4), e93474. http://dx.doi.org/10.1371/journal.pone.0093474.

Ruan, Z., Yang, Y., Zhou, Y., Wen, Y., Sheng, D., ... Yin, Y. (2014). Metabolomic analysis of amino acid and energy metabolism in rats supplemented with chlorogenic acid. http://dx.doi.org/10.1007/s00726-014-1762-7.

Sääksjärvi, K., Knekt, P., Rissanen, H., Laaksonen,M. a, Reunanen, a, \&Männistö, S. (2008). Prospective study of coffee consumption and risk of Parkinson's disease. European Journal of Clinical Nutrition, 62(7), 908-915. http://dx.doi.org/10.1038/sj.ejcn.1602788.

Scalbert, A., Brennan, L., Manach, C., Andres-Lacueva, C., Dragsted, L. O., Draper, J., ... Wishart, D. S. (2014). The food metabolome: A window over dietary exposure. The American Journal of Clinical Nutrition, 99(6), 1286-1308. http://dx.doi.org/10.3945/ajcn.113.076133.

Senn, S. (2002). Cross-over trials in clinical research. Retrieved from https://books.google.com/books?hl=es\&lr=\&id=MWbHzwpPTgEC\&pgis=1

Shukitt-Hale, B., Miller,M. G., Chu, Y. F., Lyle, B. J., \& Joseph, J. a. (2013). Coffee, but not caffeine, has positive effects on cognition and psychomotor behavior in aging. Age,35(6), 2183-2192. http://dx.doi.org/10.1007/s11357-012-9509-4.

Stadler, R. H., Varga, N., Hau, J., Arce Vera, F., \& Welti, D. H. (2002). Alkylpyridiniums. 1. Formation in model systems via thermal degradation of trigonelline. Journal of Agricultural and Food Chemistry, 50, 1192-1199. http://dx.doi.org/10.1021/jf011234k. 